\title{
SLIP VELOCITY DISTRIBUTION IN A PARALLEL PLATE CHANNEL ON ASYMMETRIC THERMAL BOUNDARY CONDITION
}

\author{
Md. Tajul Islam \\ Department of Mathematics, Begum Rokeya University, Rangpur, Bangladesh \\ Email: tajul000@yahoo.com
}

Received 13.11.2014

Accepted 28.03.2015

\begin{abstract}
Steady, laminar and fully developed flows in parallel plate microchannel with asymmetric thermal wall conditions are solved by control volume technique. In order to examine the influence of Reynolds number and Knudsen number on the velocity distributions, a series of simulations are performed for different Reynolds and Knudsen numbers. Nitrogen gas is used as working fluid and we assume the fluid as continuum but employ the slip boundary conditions on the walls. The Navier-Stokes and energy equations are solved simultaneously. The results are found in good agreement with those predicted by analytical solutions in 2D continuous flow model employing first order slip boundary conditions. It is concluded that the rarefaction flattens the velocity distribution. If the product of Reynolds numbers and Knudsen numbers is fixed, the cross sectional average velocity is fixed for incompressible flow.
\end{abstract}

Keywords: Slip, Knudsen number, Compressible, Reynolds number, Incompressible.

\section{INTRODUCTION}

Micromachining technology is a rapidly emerging technology, where new potential applications are being continuously developed. Micro-Electro-Mechanical-Systems (MEMS) applications range in a widening field of disciplines from consumer products to industrial tools. Sensors, actuators, micropumps, microvalves, microturbines etc. are the examples of some of the MEMS devices. Recently noticeable progresses have been made in design and fabrication of Micro-ElectroMechanical-Systems (MEMS). In response to the rapid progress in design and fabrication of MEMS devices, the need for understanding the momentum and heat transfer in microsystems is essential. The flows in macro and micro systems are not the same. The characteristic length scales that govern the energy and momentum transfer in MEMS and their environments are on the order of microns. Surface effects dominate in small devices. The surface-to-volume ratio for a machine with a characteristic length of $1.0 \mathrm{~m}$ is $1.0 \mathrm{~m}^{-1}$, while that for a MEMS device having a size of 1.0 $\mu m$ is $10^{-6} \mathrm{~m}^{-1}$. The millionfold increase in surface area relative to the mass of the minute device substantially affects the transport of mass, momentum, and energy through the surface. The small length scale of microdevices may invalidate the continuum approximation altogether Gad-el-Hak [13]. 
Transport phenomena at microscale reveal many features that are not observed in macroscale devices. These features are quite different for gas and liquid flows. According to Karniadakis et al. [10], four important effects are encountered in gas microflows. They are compressibility, viscous heating, thermal creep and rarefaction. Liquid flows are encountered with other microscale features such as surface tension and electrokinetic effects.

The deviation of the state of the gas from continuum behavior is measured by the Knudsen number, $K n$. For a microchannel, the Knudsen number is defined as $\mathrm{Kn}=\frac{\lambda}{D_{h}}$ where $D_{h}$ is the hydraulic diameter of the channel and for parallel plate channel it is defined by $D_{h}=2 H$.

The mean free $\lambda$ which corresponds to the distance travelled by the molecules between collisions is defined by $\lambda=\frac{k_{B} T}{\sqrt{2} \pi p \sigma^{2}}$. Consequently the Knudsen number $K n$, can be expressed as $K n=\frac{k_{B} T}{2 \sqrt{2} \pi p \sigma^{2} H}$. The local value of Knudsen number determines the degree of rarefaction and the degree of validity of the continuum model. Knudsen number is the criterion to indicate whether the flow problem can be solved by continuum approach. Flow regimes are specified according to Knudsen number as shown in Table 1.

Table 1. Different flow regimes based on Knudsen numbers.

\begin{tabular}{|l|l|l|}
\hline \multicolumn{1}{|c|}{ Regime } & \multicolumn{1}{|c|}{ Method of Calculation } & \multicolumn{1}{c|}{ Range of $\boldsymbol{K n}$} \\
\hline Continuum flow & $\begin{array}{l}\text { Navier-Stokes and energy equations with noslip boundary } \\
\text { conditions }\end{array}$ & $K n \leq 0.001$ \\
\hline Slip flow & $\begin{array}{l}\text { Navier-Stokes and energy equations with slip boundary } \\
\text { conditions }\end{array}$ & $0.001<K n \leq 0.1$ \\
\hline Transition flow & Boltzmann Transport Equations, DSMC & $0.1<K n \leq 10$ \\
\hline Free molecule flow & Boltzmann Transport Equations, DSMC & $K n>10$ \\
\hline
\end{tabular}

In the limit of zero Knudsen number, the transport term in the momentum and energy equations are negligible and the Navier-Stokes equations then reduce to the inviscid Euler equations. Both heat conduction and viscous dissipation are negligible and the flow is then approximately isentropic from the continuum viewpoint, while the corresponding molecular viewpoint is that the velocity distribution function is everywhere of the local equilibrium Gad-el-Hak [13]. As the Knudsen number increases, rarefaction effects become more important and the continuum approach breaks down for $K n>0.001$.

\section{Nomenclature}

$H \quad$ : channel height, $\mu m$

$L \quad$ : length of the channel, $\mu m$

$T$ : Temperature, $K$

$k_{B} \quad:$ Boltzmann constant

$p \quad$ : pressure, $N / m^{2}$

$K n \quad:$ Knudsen number

$R \quad$ : gas constant, $\mathrm{J} / \mathrm{kg} . \mathrm{K}$

\section{Greek symbols}

$\mu \quad$ : dynamic viscosity, N.s $/ \mathrm{m}^{2}$

$\rho \quad$ : density, $\mathrm{kg} / \mathrm{m}^{3}$

$\tau \quad:$ shear stress, $N / m^{2}$

$\sigma \quad$ : molecular diameter, Angstroms

$\sigma_{m}$ : tangential momentum accommodation coefficient

$\sigma_{m} \quad$ : thermal accommodation coefficient 
$u, v:$ velocity components, $\mathrm{m} / \mathrm{s}$

$M a$ : Mach number

Re : Reynolds number

$n \quad$ : normal coordinate on the channel wall

$x, y$ : co-ordinates distances, $\mu m$

$p_{\text {in }} \quad$ : pressure at inlet, $\mathrm{Pa}$

$p_{\text {out }}$ : pressure at outlet, $\mathrm{Pa}$

$A R$ : aspect ratio

$P R$ : pressure ratio $\lambda \quad$ : mean free path, $\mu m$

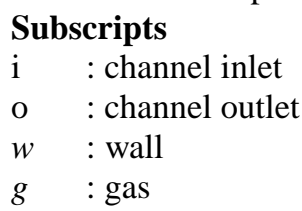

In the slip flow regime, where $0.001<K n \leq 0.1$, the fluid is not in equilibrium state in the region next to the wall which is known as Knudsen boundary layer. Consequently, there are velocity and temperature discontinuities on the walls. The Knudsen boundary layer is significant only up to a distance of one mean free path away from the wall Hadjiconstantinou [5]. Beyond the Knudsen boundary layer, the deviation from the state of equilibrium is negligible and the Navier-Stokes equations are valid there. The velocity and the temperature can be compensated by applying slip boundary conditions on the wall and then the Navier-Stokes equations and energy equations are applicable in the whole domain.

In the transition regime, where $0.1<K n \leq 10$, the molecular approach should be used or Boltzmann equation can be used directly. For higher Knudsen numbers, in the transition and free molecular flow regimes, the Boltzmann equation must be solved by appropriate numerical technique such as the Direct Simulation Monte Carlo (DSMC) methods or the Lattice Boltzmann Methods (LBM).

Fundamental work in microflows started in much earlier. In 1846, Leonard and Poiseuille [2] studied liquid flows in tubes with diameters ranging from $30 \mu \mathrm{m}$ to $150 \mu \mathrm{m}$. He focused on the relationship among flowrate, pressure drop and tube geometry. He did not consider viscosity.

The pioneer investigators in the study of gaseous flow in microchannels are Maxwell [12], Smoluchowski [18], Schaaf and Chambre [17], Pong et al. [15], Beskok and Karniadakis [1], Arkilic et al. [3], Gad-el-Hak [4].

In 1909, Knudsen [11] studied gas flow through glass capillary tubes in the transition and free molecular flow regimes. He normalized the volumetric flow rate with the inlet to exit pressure difference and plotted against the average pressure in the capillary which showed minimum at $K n=1.0$.

Sun and Faghri [6] studied the rarefaction and compressibility effects of two dimensional gaseous flow in microchannels using DSMC. They examined the effect of compressibility and rarefaction for the inlet to outlet pressure ratios ranging from 1.38 to 4.5 and Knudsen numbers ranging from 0.03 to 0.11 respectively. They reported that compressibility makes the axial pressure variation nonlinear and enhances the local friction coefficient. On the other hand, rarefaction does not affect pressure distribution but causes the flow to slip at the wall and reduces the local friction coefficient. 
Aydin and Avci [14] studied laminar forced convective heat transfer in a microchannel between two parallel plates analytically. The viscous dissipation, the velocity slip and the temperature jump at the wall are included in the analysis. Both hydrodynamically and thermally fully developed flow cases are examined. Either the hot and cold wall case is considered for the two different thermal boundary conditions, namely the constant heat flux and constant wall temperature.

Most of the studies are considered incompressible because of simplicity and low Mach number flows. There are only a few theoretical studies addressing compressible, laminar flow in uniform conduits. The studies of compressible flow, without the incorporation of rarefied behavior, have been conducted by Prud'homme et al. [16]

Prud'homme et al. [16] and H. van den Berg et al. [7] neglected the transverse velocity and used a perturbation expansion to solve the isothermal, compressible Navier-Stokes equations for laminar flow in a circular tube. For small Reynolds and Mach number flows, they obtained a 'locally selfsimilar' velocity profile.

Harley et. al. [9] conducted experimental and theoretical investigations of low Reynolds number, high subsonic Mach number, compressible gas flow in long conduits. Nitrogen, helium, and argon gases were used. The channels were microfabricated on silicon wafers. The Knudsen number ranged from $10^{-3}$ to 0.4 . The measured friction factor was in good agreement with theoretical predictions considering isothermal, locally fully developed, first order, slip flow.

The development of micromachining technology enables fabrication of micro-fluidic devices such as microvalves, micropumps, micronozzles, microsensors, microheat exchangers. Microchannels and chambers are the essential part of such devices. In addition to connecting different devices, microchannles are used for reactant delivery such as biochemical reaction chambers, in physical particle separation, in inkjet print heads, and as heat exchangers for cooling computer chips. It is already established that the flows in micro-devices behave differently from that of macro counterparts. Thus in order to design and fabricate micro-devices properly, insight characteristics of fluid flow and heat transfer in microchannels must be understood. A number of studies have been performed in this field but there are a lot of discrepancies found among the results.

The objective of the present study is to investigate the influence of Reynolds and Knudsen numbers on velocity distributions of gaseous flow in parallel plate channels. The asymmetric constant wall temperature boundary conditions are applied on the walls. To account for the noncontinuum effect, the governing equations are solved in conjunction with the slip velocity and temperature jump boundary conditions.

\section{Model Development}

\subsection{Problem Statement}

We considered nitrogen gas flow through parallel plate channels in Cartesian co-ordinates systems. Fig. 1 shows the geometry and co-ordinates of the microchannel. 


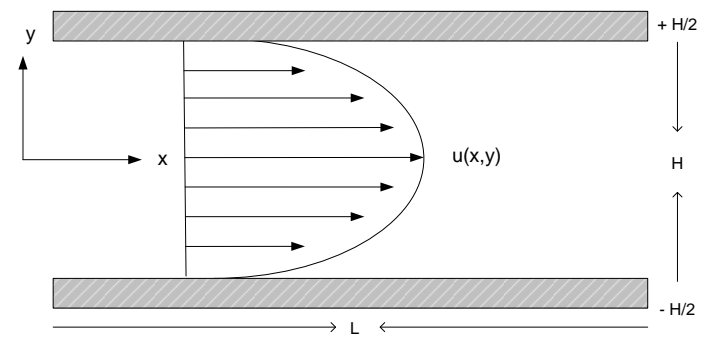

Fig. 1: Schematic diagram of the channels

The flow domain is bounded by $0 \leq x \leq L$ and $-H / 2 \leq y \leq H / 2$ and, where $L$ and $H$ are the length and height of the channels. We defined aspect ratio as $A R=\frac{L}{H}$, and pressure ratio as $P R=\frac{P_{\text {in }}}{P_{\text {out }}}$. A number of investigations are performed with slip and noslip boundary conditions on the walls. Various Reynolds and Knudsen numbers are adjusted by changing $A R$ as well as $P R$. Change of $A R$ is handled by changing $H$ for different channel heights within the range $1.25 \mu \mathrm{m} \leq \mathrm{H} \leq 5 \mu \mathrm{m}$ but keeping the channel length $L=3000 \mu \mathrm{m}$ constant. Reynolds numbers vary from 0.25 to 4.0 and the Knudsen numbers from 0.025 to 0.1 .

\subsection{Governing Equations}

The 2D gas flow and heat transfer is assumed to be steady and laminar. The Three basic laws of conservation of mass, momentum and energy are solved for both compressible and incompressible flows for Newtonian fluid. The compressible forms of the governing equations are expressed in following form and are solved with the help of state equation $p=\rho R T$.

The continuity equation:

$$
\operatorname{div}(\rho \vec{V})=0
$$

The Navier-Stokes equations:

$$
\begin{aligned}
& \operatorname{div}(\rho u \vec{V})=\frac{\partial p}{\partial x}+\operatorname{div}(\mu \text { grade } u) \\
& \operatorname{div}(\rho v \vec{V})=-\frac{\partial p}{\partial x}+\operatorname{div}(\mu \operatorname{grad} v)
\end{aligned}
$$

The energy equation:

$$
\operatorname{div}(\rho i \vec{V})=-p \operatorname{div} \vec{V}+\operatorname{div}(\kappa \operatorname{grad} T)
$$

where $i$ is the specific internal energy. The governing equations for incompressible flow are reduced from the above equations. In case of incompressible flow there is no need to establish linkage between the energy equation and the equations of continuity and momentum.

\subsection{Basic concepts}

Maxwell [12] in his study, considered the kinetic theory of dilute, monatomic gases. Gas molecules, modeled as rigid spheres, continuously strike and reflect from a solid surface, just as 
they continuously collide with each other. He assumed the surface as something between perfectly reflecting and perfectly absorbing surface. He assumed that a fraction $\sigma_{m}$ of the molecules is absorbed by the surface (due to the roughness of the wall), and then reemitted with velocities corresponding to those in still gas at the temperature of the wall. The other fraction, $1-\sigma_{m}$, of the molecules is perfectly reflected by the wall. The dimensionless coefficient $\sigma_{m}$ is called the tangential momentum accommodation coefficient. When $\sigma_{m}=0$, the tangential momentum of the incident molecules equals that of the reflected molecules and no momentum is transmitted to the wall. This kind of reflection is called specular reflection. Conversely, when $\sigma_{m}=1$, the gas molecules transmit all their tangential momentum to the wall and the reflection is a diffuse reflection. For the momentum balance at the wall, Maxwell demonstrated the slip velocity as follows:

$$
u_{\text {slip }}=u_{\text {gas }}-u_{\text {wall }}=\frac{2-\sigma_{m}}{\sigma_{m}} \lambda\left(\frac{\partial u}{\partial n}\right)_{w}+\frac{3}{4} \frac{\mu}{\rho T_{\text {gas }}}\left(\frac{\partial u}{\partial x}\right)_{w}
$$

where $\lambda$ is the mean free path and $\left(\frac{\partial u}{\partial n}\right)_{w}$ is the variation of velocity normal to wall.

In analogy with the slip phenomenon, Poisson assumed that there might be a temperature jump at the wall and proposed that the equation might be equivalent to:

$$
T_{\text {gas }}-T_{\text {wall }}=\varsigma\left(\frac{\partial T}{\partial n}\right)_{w}
$$

where $\varsigma$ is the temperature jump distance. Smoluchowski [18] experimentally confirmed the hypothesis and suggested that $\varsigma$ is proportional to the mean free path $\lambda$. Like slip velocity, a fraction $\sigma_{T}$ of the molecules come in contact with the walls and the walls adjust their mean thermal energy. These molecules are reflected from the walls with the temperature of the gas on the walls. The other fraction $1-\sigma_{T}$ of the molecules reflects with their incident thermal energy. The dimensionless coefficient $\sigma_{T}$ is called the thermal accommodation coefficient. For an energy balance on the walls, Smoluchowski demonstrated the temperature jump as follows:

$$
T_{\text {gas }}-T_{\text {wall }}=\left(\frac{2-\sigma_{T}}{\sigma_{T}}\right) \frac{2 \gamma}{\gamma+1} \frac{\lambda}{\operatorname{Pr}}\left(\frac{\partial T}{\partial n}\right)_{w}
$$

Where $\operatorname{Pr}=\frac{\mu c_{p}}{k}$ is the Prandtl number.

\subsection{Boundary Conditions}

Asymmetric wall thermal condition is implied on the walls. The free stream temperature and the lower wall temperature are kept at the same constant temperature which is lower than that of the upper wall and the difference between the upper and lower wall temperature varies from $100 \mathrm{~K}$ to $300 K$. Pressure condition is assumed on the outlet boundary and velocity inlet and pressure 
condition are implied on the inlet boundary according to incompressible and compressible flow respectively.

We impose slip velocity and temperature jump boundary conditions on the walls. The slip velocity condition was proposed by Maxwell [12] as

$$
u_{\text {gas }}-u_{\text {wall }}=\frac{2-\sigma_{m}}{\sigma_{m}} K n \frac{\partial u}{\partial n}
$$

and the temperature jump boundary conditions by Smoluchowski [18] as

$$
T_{\text {gas }}-T_{\text {wall }}=\left(\frac{2-\sigma_{T}}{\sigma_{T}}\right) \frac{2 \gamma}{\gamma+1} \frac{\lambda}{\operatorname{Pr}}\left(\frac{\partial T}{\partial n}\right)_{w}
$$

where $\frac{\partial u}{\partial n}$ and $\frac{\partial T}{\partial n}$ are the variation of velocity and temperature normal to the wall. We consider the tangential-momentum-accommodation coefficient $\sigma_{m}=1$ and the thermal-accommodation coefficient $\sigma_{T}=1$ which describe the gas-wall interactions.

\subsection{Numerical Method}

The control volume method is used to discretize the governing equations. The pressure based segregated solver is employed in order to achieve steady state analysis. The momentum and energy equations are solved with second-order up-wind scheme to interpolate the corresponding cell center variables to the faces of the cells. The SIMPLE (Semi-Implicit Method for Pressure-Linked Equations) algorithm is used for introducing pressure into the continuity equation. A second-order pressure interpolation method is used to calculate the pressure at cell faces from the neighboring nodes. The computations are considered to be converged when the residues for continuity, momentum and energy are less than $10^{-6}$.

\subsection{Grid dependency Test}

To evaluate the grid size effect, grid dependency tests are carried out. Three different sizes of grid $30 \times 1200,40 \times 1500$ and $50 \times 1800$ are tested for a typical channel of $A R=600$ for incompressible flow with slip boundary conditions and the results are listed in Table 2 . The table shows that the relative difference of average velocity at outlet for grid sizes $30 \times 1200$ and $40 \times$ 1500 is $0.27 \%$ and for grid sizes $40 \times 1500$ and $50 \times 1800$ is $0.04 \%$ respectively. For convenience, we used grid size $40 \times 1500$ or its multiple according to the length of the dimensions of the domain.

Table 2. Grid dependence test of the average velocity under three sizes of grids

\begin{tabular}{|c|c|}
\hline Grid size & Average velocity \\
\hline $30 \times 1200$ & 19.49037 \\
\hline $40 \times 1500$ & 19.54263 \\
\hline $50 \times 1800$ & 19.55102 \\
\hline
\end{tabular}




\subsection{Results and Discussion}

In order to validate our model we simulated $2 \mathrm{D}$ isothermal flow with first order slip boundary conditions. Nitrogen gas was used with channel $A R=2500$ and $P R=2.36$. The outlet Reynolds and Knudsen numbers were 1.99 and 0.044 respectively. The Centerline velocity distribution normalized with the speed of sound at the inlet is compared with Graur at el. [8] those predicted by analytical solutions derived from the quasi gas dynamic equations with the same conditions. The results are displayed in Fig. 2. The square symbols represent the analytical results and the solid line represents the results from current work. The results show good agreement which validate our model.

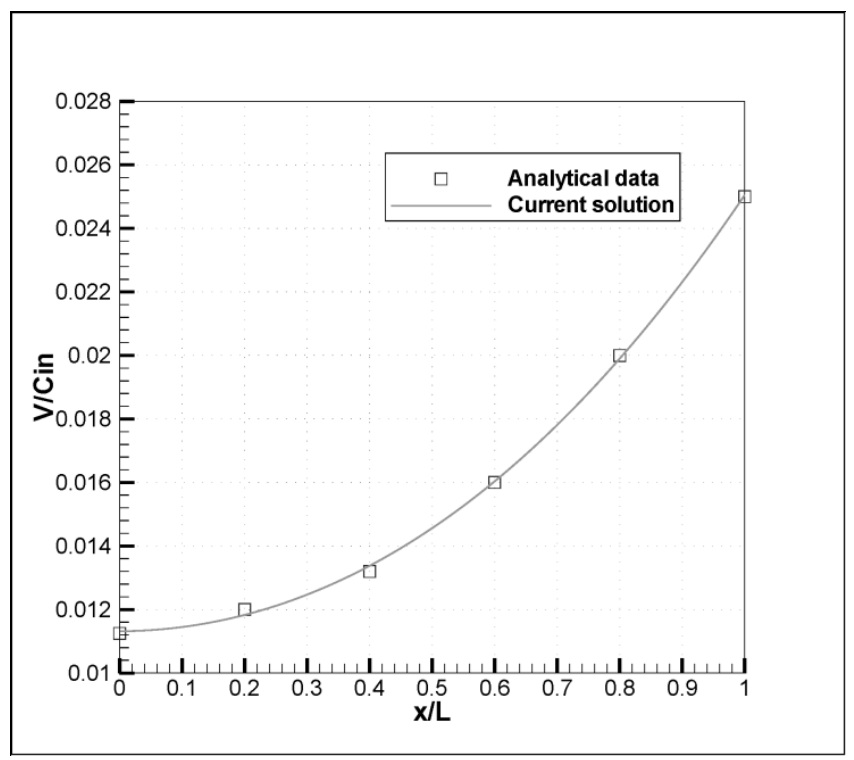

Fig. 2: Centerline velocity distribution normalized with the speed of sound at the inlet.

In our study, Reynolds number $R e$ and Knudsen number $K n$ are the main parameters of our investigation. We study the interactive effects of these parameters on flow velocity distribution for the fully developed flow.

We examine the case of asymmetric thermal boundary condition with constant temperature at the walls. When the upper wall temperature are kept at $300 \mathrm{~K}$ and the lower wall and the free stream temperature are kept at $200 \mathrm{~K}$, the condition is denoted by Tup 300 and when the upper wall and the free stream temperature are kept at $200 \mathrm{~K}$ and the lower wall temperature are kept at $300 \mathrm{~K}$, it is denoted by Tup200 in the present article. Slip velocity and temperature jump boundary conditions are applied on the walls. Our investigation covers both the compressible and incompressible flow. The velocity distributions of incompressible flow are discussed by the figures from Fig. 2 to Fig.5 and those of compressible flow by the figures from Fig. 6 to Fig. 8 . 


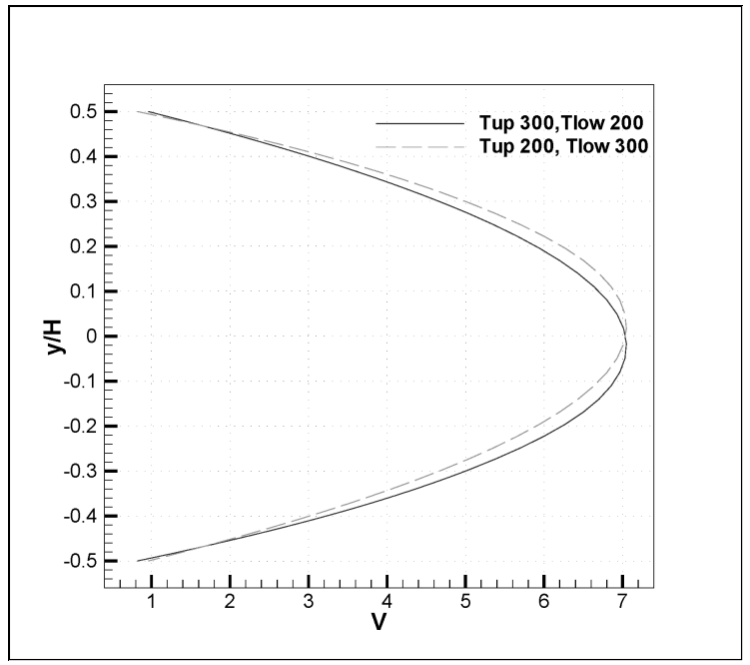

Fig. 3: Cross sectional velocity distributions with asymmetric wall thermal conditions for incompressible flow.

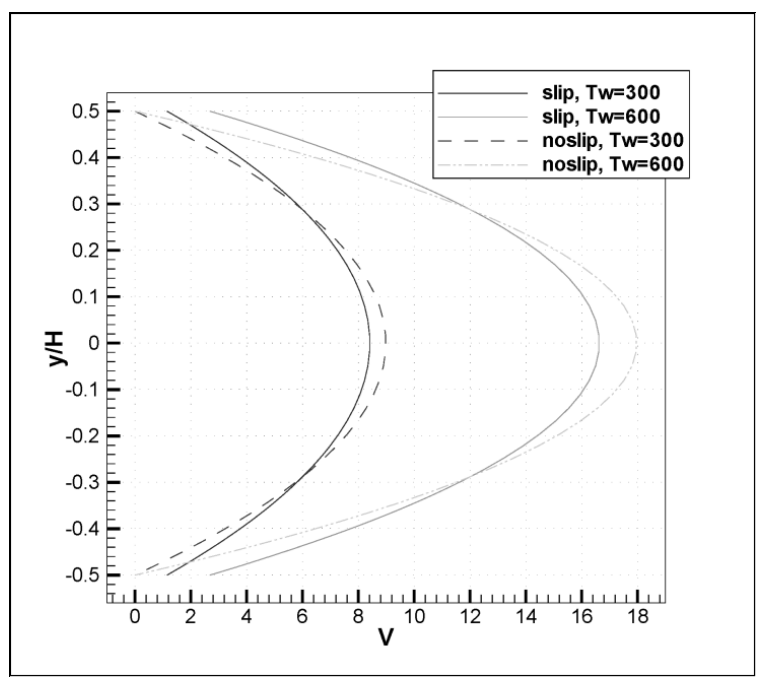

Fig. 4: Cross sectional velocity distributions with slip and noslip conditions for incompressible flow.

The cross sectional velocity distributions with slip boundary conditions of incompressible flow are shown in Fig. 3. For Tup300 condition Knudsen number on the upper wall is 0.024 and that on the lower wall is 0.017 . Consequently the fluid velocity on the upper wall is higher than that of the lower wall which is 0.96 and 0.82 on the upper and lower wall respectively. Since the flow is incompressible, the cross sectional average velocity will remain the same. As a result the velocity of the fluid near the upper wall will be lower than the velocity of the corresponding fluid near the lower wall. For Tup200 condition we see the opposite scenario which is displayed by the Fig. 3.

Then we consider two cases of symmetric thermal boundary conditions at the walls. In the first case the upper and lower wall temperature are kept at the same constant temperature $300 \mathrm{~K}$ with 
slip and noslip boundary conditions. In the second case the wall temperature $300 \mathrm{~K}$ is increased to $600 \mathrm{~K}$ remaining all other conditions the same. The cross sectional velocity distributions for slip and noslip boundary conditions for two cases of wall temperature are displayed in Fig. 4.

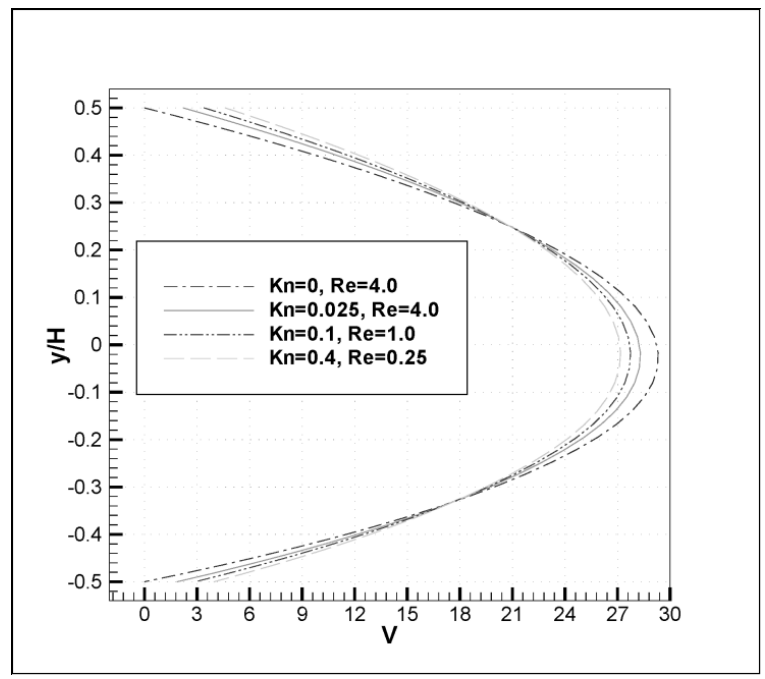

Fig. 5: Velocity profiles at $x / L=0.9$ with different Knudsen and Reynolds numbers for incompressible flow.

At $300 \mathrm{~K}$ wall temperature the outlet Reynolds number is 0.88 for both slip and noslip conditions and the outlet Knudsen number is 0.029 for slip condition. At temperature $600 \mathrm{~K}$ the corresponding Reynolds and Knudsen numbers are 0.53 and 0.059 respectively. We see that for slip condition there is slip on the wall and the fluid velocity near the wall is higher than that of noslip condition. But near the core region the fluid velocity with noslip boundary condition is higher than that of slip condition. As a result the average outlet velocities for both the flows with slip and noslip boundary conditions are equal and fixed. At temperature $300 \mathrm{~K}$ the average velocity for slip and noslip condition at the outlet is 5.99 which increases to 11.99 for the increase of temperature from $300 \mathrm{~K}$ to $600 K$.

Table 3. Average outlet velocity and mass flow rate for incompressible flow.

\begin{tabular}{|c|c|c|c|c|}
\hline $\mathrm{Re}$ & $\mathrm{Kn}$ & Re.Kn & Outlet average velocity & Mass flow rate \\
\hline 4.0 & 0.025 & 0.1 & 19.56 & $3.12 \mathrm{e}-05$ \\
\hline 1.0 & 0.1 & 0.1 & 19.56 & $7.81 \mathrm{e}-06$ \\
\hline 0.25 & 0.4 & 0.1 & 19.56 & $1.95 \mathrm{e}-06$ \\
\hline
\end{tabular}

To examine the simultaneous effect of compressibility and rarefaction that is Reynolds and Knudsen numbers on flow properties we investigated four cases with different sets of combinations of Reynolds and Knudsen numbers. Among the four cases, the first one is with noslip boundary condition and the rest are with slip boundary condition. The results are depicted by Fig. 5. From the figure we see that for noslip condition fluid velocity on the wall is zero. As the rarefaction increases, fluid velocity on the wall increases while the maximum velocity decreases. Consequently the outlet average velocities for all the slip and noslip wall conditions are equal 
which is $19.58(\mathrm{~m} / \mathrm{s})$. All the velocity distributions shown in Fig. 5, intersect at a fixed point. Here the product of Reynolds number and Knudsen number for the slip wall conditions is 0.1 . The Table 3 shows the outlet average velocity and mass flow rate for different Reynolds and Knudsen numbers.

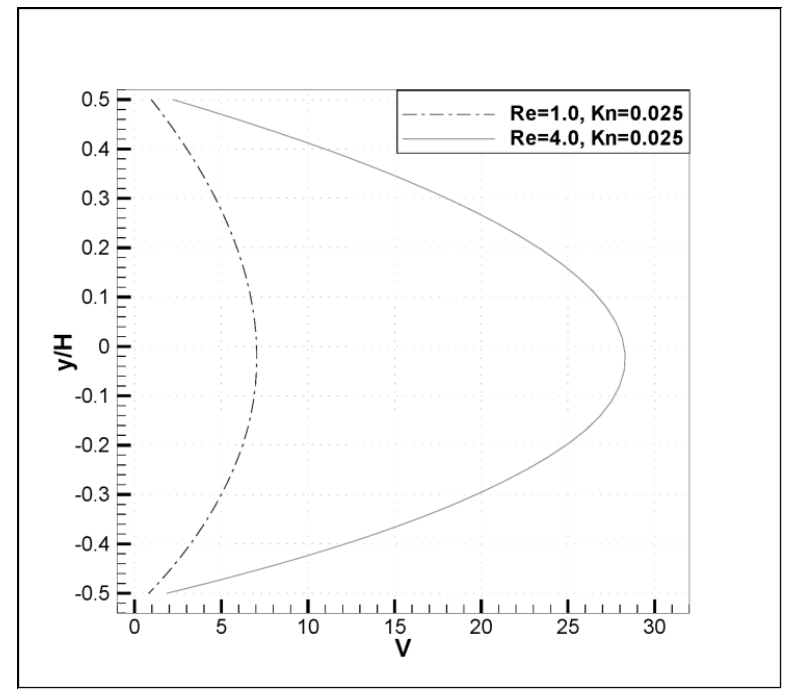

Fig. 6: Velocity distributions for incompressible flow with fixed Knudsen number but different Reynolds numbers.

The effects of Reynolds number on velocity are shown in Fig. 6. The cross sectional velocity distributions are depicted for fixed Knudsen number but varying Reynolds numbers. The Knudsen number is fixed to 0.025 while the Reynolds numbers vary from 1.0 to 4.0 . For this variation of Reynolds number the average outlet velocity increases from 4.99 to 19.55 .

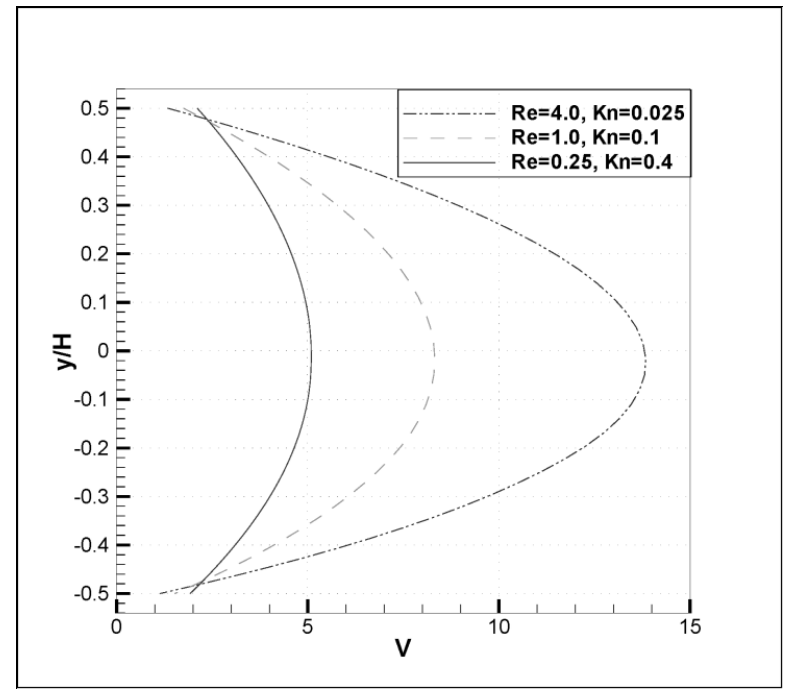

Fig. 7: Velocity profiles with different Knudsen and Reynolds numbers for compressible flow. 
Velocity distributions for different Reynolds and Knudsen numbers for compressible flow are displayed in Fig. 7. Here the product of the Reynolds number and the Knudsen number are fixed to 0.1. A similar velocity distribution with the same Reynolds and Knudsen numbers is displayed for incompressible flow in Fig. 5. From Fig. 7 we see that the velocity distributions intersect at a fixed points but the outlet average velocity are not equal as it does for incompressible flow.

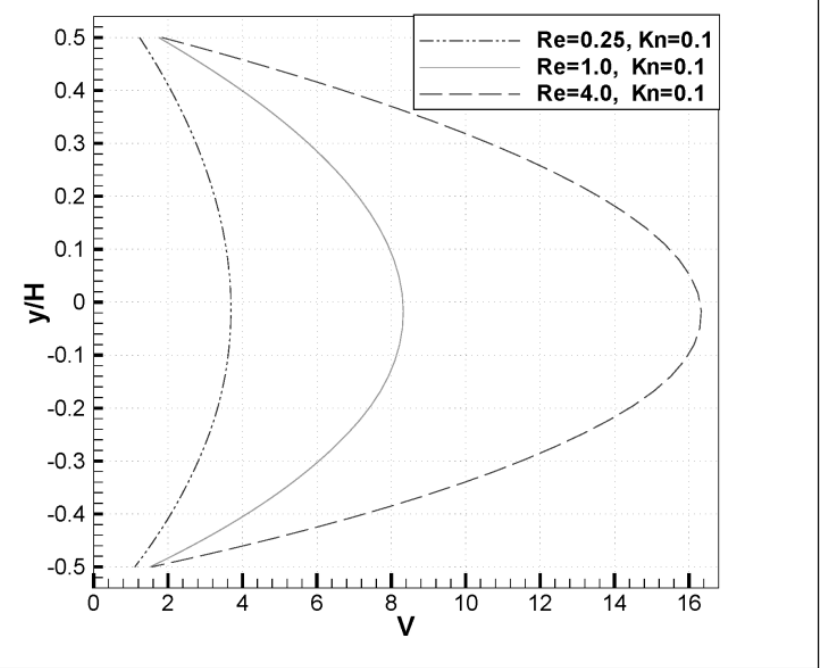

Fig. 8: Velocity distribution with fixed Knudsen numbers but varying Reynolds numbers for compressible flow.

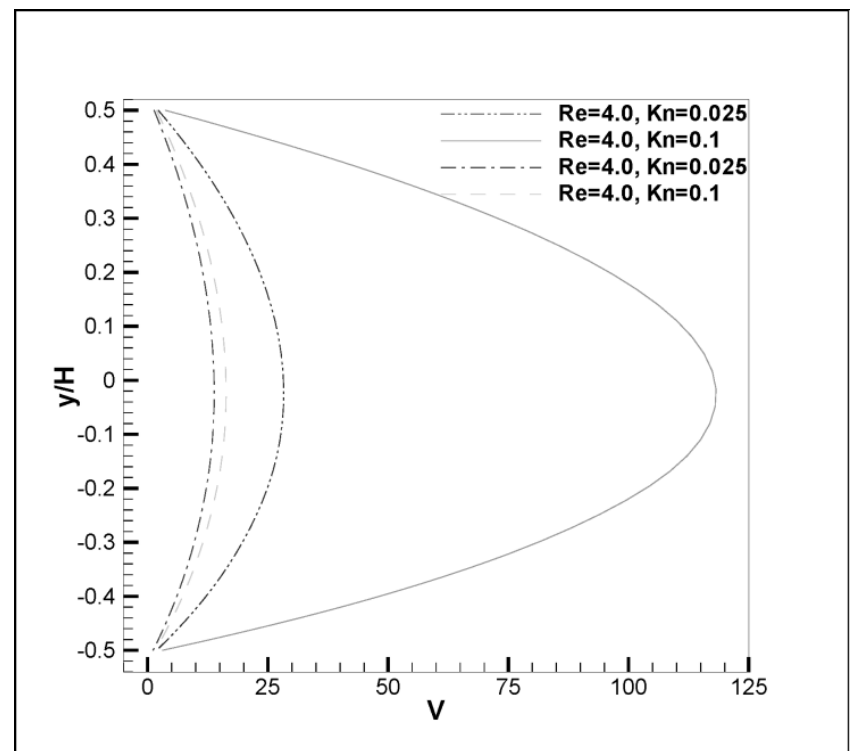

Fig. 9: Velocity distribution at with different Knudsen numbers for compressible and incompressible flow. Curve without symbol is for compressible flow, Curve with symbol is for incompressible flow. 
Fig. 8 shows that cross sectional velocity distribution for compressible flow for different Reynolds numbers. We kept the Knudsen number fixed to 0.1 and vary Reynolds numbers from 0.25 to 4.0. The fluid velocity on the wall for Reynolds numbers $0.25,1.0$ and 4.0 are $1.23,1.75$ and 1.84 and the corresponding outlet average velocity are 4.96, 20.12 and 78.39 respectively. We see that for the increase of Reynolds numbers from 0.25 to 1.0 , the slip velocity on the wall increases $42.28 \%$ and the outlet average velocity increases 4.06 times. Again for the increase of Reynolds numbers from 1.0 to 4.0 the slip velocity on the wall increases $5.14 \%$ and the outlet average velocity increases 3.90 times. It can be concluded that the rate of increase of wall slip velocity as well as outlet average velocity decreases for the increase of Reynolds numbers. This may be due to the effect of compressibility.

Fig. 9 shows the comparison of velocity distribution for compressible and incompressible flow at fixed Reynolds but different Knudsen numbers. The figure shows that for the same combination of Reynolds and Knudsen numbers, the velocity for incompressible flow is higher than that of the compressible flow.

The gas velocities on the wall for compressible and incompressible flow are furnished in the Table 4.

Table 4. Slip velocity on the walls for compressible and incompressible flows.

\begin{tabular}{|c|c|c|c|}
\hline & & \multicolumn{2}{|c|}{ Gas velocity on the wall } \\
\hline$R e$ & $K n$ & Incompressible flow & Compressible flow \\
\hline 4.0 & 0.025 & 2.20 & 1.34 \\
\hline 1.0 & 0.1 & 3.40 & 1.75 \\
\hline 4.0 & 0.1 & 3.66 & 1.84 \\
\hline 0.25 & 0.4 & 4.60 & 2.11 \\
\hline
\end{tabular}

The table shows that for the same Reynolds and Knudsen numbers, the fluid velocity on the wall for incompressible flow is higher than that of the compressible flow. The gas velocity on the wall increases 4.08 times for incompressible flow and 4.02 times for compressible flow. Here the product of Reynolds and Knudsen numbers are kept 0.1 .

\section{Conclusions}

The effect of slip flattens the velocity distribution relative to that for noslip flow. Temperature increases the slip velocity on the wall and decreases the maximum velocity and consequently the velocity distributions get flattened. If the velocity distribution is the function of Reynolds and Knudsen numbers and if their product is fixed then the corresponding average velocity for incompressible flow is fixed but for compressible flow it varies. For the same Reynolds and Knudsen numbers the velocity for incompressible flow is higher than that of the compressible flow counterpart. 


\section{REFERENCES}

[1] Ali Beskok and George E. Karniadakis, "Simulation of Heat and Momentum Transfer in Complex Microgeometries", Journal of Thermophysics and Heat Transfer, vol. 8, no.4, pp. 647-655, 1994.

[2] Dr Jean Leonard Marie Poiseuille, "Experimental investigations upon the flow of liquids in tubes of very small diameter", Lancaster Press Inc., Lancaster, vol. 1, no. 1, 1846.

[3] Errol B. Arkilic, Kenneth S. Bueuer, and Martin A. Schmidt, "Gaseous Slip Flow in Long Microchannles", Application of Microfabrication to Fluid Mechanics", ASME, vol. 197, pp. 57-65, 1994.

[4] Gad-el-Hak, M., "The fluid mechanics of microdevices - The Freeman Scholar Lecture", J. Fluids Eng., vol. 121, pp. 5-33, 1999.

[5] Hadjiconstantinou, N.G., "The limits of Navier Stokes theory and kinetic extensions for describing small scale gaseous hydrodynamics". Phys. Fluids, vol. 18, pp. 1070-6631, 2006.

[6] Hongwei Sun and Mohammad Faghri, "Effects of Rarefaction and Compressibility of Gaseous Flow in Microchannel using DSMC", Numerical Heat Transfer, Part A, 38, pp.153-168, 2000.

[7] H. van den Berg, C. Seldam, and P.Gulik, "Compressible Laminar flow in a capillary", J. Fluid mech., vol. 246, pp. 1-20, 1993.

[8] I. A. Graur, J. G. Meolans, D. E. Zeitoun, "Analytical and numerical descriotion for isothermal gas flows in microchannels", Microfluidics and Nanofluidics, vol. 2, pp. 64-77, 2006.

[9] John C. Harley, Yufeng Huang, Haim H. Bau and Jay N. Zemel, "Gas flow in micro-channels", J. Fluid Mech., vol. 284, pp. 251-274, 1995.

[10] Karniadakis, G., Beskok, A. \& Aluru, N., Microflows and Nanoflows, Fundamentals and Simulation, Springer, New York, 2005.

[11] Knudsen, M., "Die gesetze der molekularstrmung und der inneren reibungsstrmung der gase durchrhren", Annalen der Physik, vol. 28, pp. 75-130, 1909.

[12] Maxwell, J.C., "On Stresses in Rarified Gases Arising From Inequalities of Temperature”, Philos. Trans. R. Soc. London, vol. 170, pp. 231-256, 1879.

[13] Mohamed Gad-el-Hak, The MEMS Handbook ( $2^{\text {nd }}$ edition), CRC press, New York, 2006.

[14] Orhan Aydin and Mete Avci, "Analysis of laminar heat transfer in micro-Poiseille flow", International Journal of Thermal Sciences, vol.46, pp. 30-37, 2007.

[15] Pong, K. C., Ho, C. M. Liu, J. and Tai, Y. C., "Non-linear Pressure Distribution in Uniform Microchannels", Application of Microfabrication to Fluid Mechanics, ASME, vol. 197, pp. 51-56, 1994.

[16] R. Prud'homme, T. Chapman, and J. Bowen, "Laminar Compressible Flow in a Tube", Appl. Sci. Res., vol. 43, pp. 67-74, 1986.

[17] Schaaf, S. and Chambre, P., Flow of Rarefied Gases ( $1^{\text {st }}$ edition), Princeton University Press, Princeton, 1961.

[18] Smoluchowski, M., "Über den Temperatursprung bei Wärmeleitung in Gasen.”, Akad. Wiss. Wien. CVII, pp. 304-329, 1898. 